\title{
Déclinaison sur la recomposition des territoires dans le cadre français
}

Thougts on the recomposition of territories in the French context

Überlegungen zur territorialen Neuordnung der Territorien im französischen

Rahmen

Henri Nonn

\section{(2) OpenEdition}

\section{Journals}

Édition électronique

URL : http://journals.openedition.org/rge/4031

DOI : $10.4000 /$ rge.4031

ISSN : 2108-6478

Éditeur

Association des géographes de l'Est

Édition imprimée

Date de publication : 1 septembre 2000

ISSN : 0035-3213

Référence électronique

Henri Nonn, "Déclinaison sur la recomposition des territoires dans le cadre français », Revue Géographique de l'Est [En ligne], vol. 40 / 4 | 2000, mis en ligne le 26 juillet 2013, consulté le 08 septembre 2020. URL : http://journals.openedition.org/rge/4031 ; DOI : https://doi.org/10.4000/rge. 4031

Ce document a été généré automatiquement le 8 septembre 2020

Tous droits réservés 


\title{
Déclinaison sur la recomposition des territoires dans le cadre français
}

\author{
Thougts on the recomposition of territories in the French context \\ Überlegungen zur territorialen Neuordnung der Territorien im französischen \\ Rahmen
}

Henri Nonn

1 On ne saurait en quelques pages entrer très avant dans la complexité de définitions des territoires, ni dans l'épistémologie consacrée à leurs recompositions. La littérature, géographique et autre (histoire, administration et management publics, droit et sciences politiques développement local...) est d'ailleurs abondante sur ces sujets ${ }^{1}$. Chaque discipline isolément, ne peut en outre s'approprier ces concepts, qui gagnent à être approchés dans l'interdisciplinarité 2 . La diversité des contenus, des organisations des territoires et des dynamiques intervenantes dans les recompositions, engage à la saisie des interdépendances y opérant. Aussi, ces lignes se veulent modestes et sans a priori. Si ce n'est celui de privilégier l'idée que les territoires sont des « organisations socio-spatiales vivantes », des « lieux de liens » (J.-L. Guigou, 1995), exprimés par des cohérences, des cohésions, des consciences d'appartenance (voire d'appropriation), des solidarités collectives (dans la vie, l'action, les projets); et qu'ils sont en continuels ajustements de leur "système", en général ouvert - plus ou moins - sur les « environnements " qui les influencent. La « recomposition ", dès lors, refléterait les cas de transformations les plus fortes (jusqu'à la rupture parfois), s'accompagnant de modifications des systèmes d'acteurs, d'échelles spatiales, de rapports entre les processus (endogènes, exogènes) qui les animent.

Dans les choix, on a ici limité le regard à ce qui relève de l'économique, du social et de l'institutionnel, transcrits territorialement, et à leurs déclinaisons dans le cadre français, ainsi qu'à quelques grands moments dans l'époque contemporaine et à des questionnements sur les recompositions en cours. 


\section{Autour de deux grands « moments " de recompositions auX XIX-XXe siècles}

3 S'y arrêter quelque peu sert à établir une «mémoire » et des héritages marquant encore les mutations actuelles.

\section{A. A la charnière entre « France rurale » et « France industrielle et urbaine »}

Bien des territoires, au début du XIXe siècle, gardaient des structurations rurales, en juxtaposition davantage qu'en articulation, avec quelques grandes familles typologiques cependant. Les « campagnes agricoles traditionnelles » se signalaient par leurs cadrages étroits aux milieux naturels (climats, reliefs, sols) donnant potentialités et contraintes, et aux systèmes agraires, aux structures démographiques et sociales : en découlaient les densités permises, des " fragilités d'équilibre » (émigrations, sensibilité aux aléas), des « centrages » très liés aux organisations sociales locales, aux marchés de proximité et aux pôles locaux de services, d'autant plus nets que les accessibilités vers l'extérieur étaient restreintes ${ }^{3}$. D'autres, en revanche, étaient précocement "ouvertes", en fonction de stimulations fournies par des axes majeurs d'échanges à grande distance et par des villes négociantes ou pourvoyeuses de travail, de marchés, de capitaux, etc. D'autres encore, à la faveur de ressources locales, de savoir-faire acquis et entretenus, constituaient des "campagnes industrieuses ». Systèmes locaux, bases économiques et de circulation, champs rationnels et de diffusion (techniques, idées...) primaient sur les constitutions de hiérarchies urbaines (faibles) et d'administration ou services (limités) dans l'animation des territoires où les proximités sociales et géographiques et les notabilités locales structuraient les relations.

D'importantes recompositions interviennent alors par la conjonction de plusieurs «révolutions». Le développement des transports modernes tend à susciter la constitution de grandes régions d'agriculture (s.l.) spécialisées : céréalières, viticoles, d'élevage, maraîchères, tournées vers des marchés nationaux, voire plus vastes, déclassant encore davantage des campagnes «traditionnelles ». Dans celles-ci, et celles dont les bases industrieuses étaient modestes ou très concurrencées, routes et chemins de fer contribuent aux «ruptures» de leur peuplement et de leurs "équilibres fragiles ». L'essor industriel vient inscrire les bassins d'industries lourdes ou diffuses (textile, mécanique) en surimposition sur les pays ruraux préexistants (travail, genres de vie mixtes); il étoffe en activités manufacturières et négociantes les villes les mieux desservies en transports, disposant de capitaux et de services. La dissociation entre production et vente, via les grossistes - collecteurs, redistributeurs - et le commerce de détail, valorise les villes-carrefours, intègre les campagnes plus accessibles à des armatures urbaines qui se hiérarchisent (nodalités, attractivité, concentrations économiques). Ce à quoi contribue également l'affirmation des encadrements administratifs (départements, cantons) dont les cadres infléchissent les anciens territoires et où les chefs-lieux gagnent en centralités publiques et privées s'additionnant.

6 Les interdépendances de ces facteurs jouent ainsi sur les trames territoriales, leurs bases économiques et sociales (diversification des activités, des emplois, des flux migratoires et relationnels), les modes de vie...; elles affectent certains éléments des 
cohésions traditionnelles, et "surlignent" les organisations institutionnelles et économiques, les cohérences " fonctionnelles ", changent les horizons des réseaux. Les «variables anthropologiques et historiques» dégagées par E. Todd (in : L'invention de l'Europe, 1990 et 1996, éd. Seuil) modulent elles aussi spatialement, par leurs charges humaines et socio-culturelles, les modalités de diffusion des "progrès ». Tandis que l'affirmation du «système national» (initiative et gestion publiques, capitalisme et patronat nationaux, équipements du territoire) se répercute sur des moyens endogènes régionaux et locaux toujours plus inscrits dans un modèle "centre-périphéries " à facettes multiples et imbriquées. Il faudra beaucoup de temps et d'énergies pour repositionner sur d'autres fondements le développement des territoires...

\section{B. La remise en cause des organisations dans la 2e moitié du XXe siècle}

7 Les prolongements sur un siècle environ des mutations précédentes ont engendré, quoique avec des ajustements progressifs, des marquages profonds dont les remises en question se cumulent autour des années 1970-90.

\section{Dans l'économie, les concentrations industrielles et financières et l'affermissement du modèle fordiste génèrent des dispositifs « centres- périphéries »}

8 Aux premiers reviennent le commandement, l'organisation, les fonctions nobles d'entreprise ; aux secondes, les usines de production, d'exécution; ces dernières sont mobilisées spatialement, via les villes moyennes et petites ou les bourgs jusque dans les campagnes, lorsqu'elles sont bien desservies en transports et/ou disposent de maind'œuvre et de savoir-faire. Et, après la seconde guerre mondiale, la diffusion des ateliers par les grandes firmes nationales, par les investissements étrangers, par des politiques de desserrement (agglomération parisienne surtout), y sollicitent les actifs - et des PME par la sous-traitance - là où le développement de l'agriculture productiviste comme la croissance démographique changent les données de l'emploi. La "dépendance " devient ainsi le lot de beaucoup de territoires mis aussi dans l'influence des villes dispensatrices des services supérieurs et d'intermédiation économique. L'inégal développement économique, dans ces conditions, n'est encore qu'assez peu corrigé, malgré des politiques volontaristes d'incitations et d'équipements à l'échelle nationale.

9 Subsistent des « régions non métropolisées » (B. Kayser), des «marges », des « zones rurales fragiles » (Datar), des «zones d'ombre » (déclins démographique et d'activités comme d'emploi)... que les aides aux aménagements techniques ou fonciers, à la "rénovation rurale», à "l'économie montagnarde »... transforment aussi « du dedans » sur d'autres volets que ceux de l'économie locale. S'élargissent par ailleurs les territoires inclus dans les «zones de peuplement industriel et urbain» (ZPIU), satellisés, liés aux agglomérations par les migrations pendulaires et les services urbains. Tandis que la contraction de quelques grandes branches industrielles concurrencées affecte les territoires dont celles-ci étaient des secteurs d'emploi et de ressources dominants (en reconversion).

10 Avec l'internationalisation de l'économie et de la concurrence, les chocs pétroliers, les crises affectant des branches entières d'industrie répercutées sur l'emploi, d'une part, 
avec la tertiarisation d'autre part, de nouvelles donnes s'établissent pour les systèmes productifs et les marchés. Dans la compétition, la logistique l'emporte sur les proximités ; la performance repose sur l'organisation, l'innovation et la technologie, les réseaux, la réactivité-flexibilité plus que sur la disponibilité de main-d'œuvre et de savoir-faire; sont valorisés les logiques "assurantielles» (= recherche du moindre risque) et les "potentiels extra-économiques» (P. Veltz, 1996). Le «modèle métropolitain» devient essentiel comme processus de développement ${ }^{4}$. Il induit maintes mutations sur les organisations territoriales et sociales (destructurations, recompositions, brouillages...) dans les aires métropolisées et par-delà, selon les distances au pôle moteur, ses axes et relais d'expansion, ou selon les capacités de "résistances locales ", la nature même des bases de métropolité et leur puissance. Les autres assiettes de développement territorialisé en «districts», «milieux innovateurs ", "systèmes productifs locaux" associent davantage technologie et cohésions résiliaires d'ordre social qui entrent également pour signaler «les régions qui gagnent» ou les "espaces régionaux actifs » ${ }^{5}$. De même, la voie étroite du développement local suppose des forces endogènes convergentes, articulées à la fois entre elles et avec l'extérieur, d'autant plus que des «seuils» minimaux d'éléments mobilisables sur cet objectif doivent être atteints (peuvent-ils l'être dans le cadre des petites intercommunalités de base?

\section{Le centralisme institutionnel national s'est, après 1945, renforcé avec la multiplication des tâches de l'Etat (planification, aménagement-équipement du territoire, rôle social...).}

11 Les collectivités locales n'ont alors que d'assez modestes capacités d'initiative (cantonnées sur la gestion locale des circonscriptions et de leurs habitants), dépendantes souvent des subventions nationales. Dans le même temps, l'action publique de développement crée de «nouveaux territoires»: ceux des zonages et périmètres d'intervention et d'aide, fréquemment propres à chaque champ sectoriel ${ }^{6}$; les premières "globalisations" concernent les SDAU (schémas directeurs) et les schémas de massifs (montagneux).

12 Mais l'accroissement des charges de l'Etat - et la dispersion de ses moyens - d'une part, la «montée » du local et du régional d'autre part, conduisent par étapes à la déconcentration administrative (1964), puis à la décentralisation et aux contractualisations (a/c.1982/3) comme aux cofinancements. Les «territoires institutionnels» des collectivités locales (Régions, départements, villes, EPCI) s'affirment, ainsi que le rôle de médiateurs de leurs élus. Reste que des différenciations ressortent : à travers les degrés de dépendance des flux d'argent public ${ }^{7}$; en fonction des situations géographiques vis-à-vis d'un contexte plus européen (investissements productifs, infrastructures, coopération); selon les capacités managériales d'ingénérie territoriale et de synergie des acteurs en régions, etc. Le " mécano institutionnel » (R. Hertzog), quoique complexifié, devient une dimension forte dans la recomposition territoriale. 
3. Des changements sociétaux et comportementaux touchent les systèmes sociospatiaux.

13 La démographie, après le "baby boom ", évolue vers une baisse sensible de la fécondité : les femmes sont plus nombreuses sur le marché du travail; les études s'allongent; le temps libre et la consommation des services s'étendent. Les changements résidentiels, par choix (périurbanisation, attractivité urbaine ou rurale) ou par obligation (emploi, formation) et l'immigration brassent plus fortement les populations (au risque de tensions...) pendant que les mobilités sont facilitées par la motorisation des ménages, les désenclavements ou améliorations d'accessibilités. Structures familiales et de proximité comme réseaux sociaux se transforment: avec plus d'individualisme; des pratiques territoriales courantes vécues à plusieurs échelles ; baisse de recours aux équipements, commerces et services locaux «banaux "; mais aussi nouvelles "valeurs» investies: lieux identitaires, patrimoine, nature et cadre de vie-environnement...

Ces diverses mutations accusent désormais ou créent des césures entre et dans les territoires : entre campagnes dévitalisées, marges, et axes ou aires métropolisés ; au sein des espaces régionaux et des armatures urbaines; à l'intérieur des territoires écartelés ou sous dépendances des aires urbaines, entre anciennes et nouvelles campagnes (B. Kayser), etc. ${ }^{8}$

\section{Actuellement : forces et faiblesses des territoires, opportunités, menaces et interrogations ${ }^{9}$}

Si on a initialement rappelé les territorialisations de la «France rurale» (en songeant aux « pays » que la géographie a longtemps décrits) et les processus majeurs survenus depuis, c'est pour dégager les complexités additionnées conduisant à la diversité des constats actuels d'appréciation. Celle-ci met en avant des critères définis par plusieurs travaux géographiques récents ${ }^{10}$, et avancés de manière très générale par la LOADT de 1995 à propos des "pays »: les territoires se définissent par leur cohésion "socioéconomico-spatiale » et une certaine communauté d'intérêt, toutes deux porteuses de projet. Ce qui s'énonce pour les "pays» peut d'ailleurs s'exprimer pour d'autres échelles : de celle du «bassin de vie » (chartes d'aménagement et de développement) à celle de l'espace régional (autour des contrats de plan, des plans régionaux, des schémas de services, d'aménagement ou de prospective).

\section{A. Les éléments de cohésion ont été bien cernés par C. Rolland-May (citée en note 10) que nous reprenons ici :}

16 a) rassemblement d'entités élémentaires solidaires et de forces et énergies endogènes suffisantes pour résister aux "forces dissipatives" (internes ou externes) qui désorganiseraient, fragmenteraient le territoire. Une histoire ou un destin communs, une cohérence géographique ou économique, une culture héritée peuvent créer des parentés assez nettes pour prévaloir sur un sentiment de diversité affaiblissante, nourrir une identité y contribuant, mais à elle seule insuffisante aujourd'hui. 
17 b) réalisation d'une "logique d'ensemble ", d'un «ordre spatial » (avec rôle éminent tenu par «l'organisation du réseau urbain » : centralités, alliances) à même de réagir aux conditions actuelles où « tout bouge et tout change » (J. Renard, ibid.).

c) émergence de projet(s) collectif(s), vecteur(s) d'éléments identitaires et d'organisation tournés vers l'intérêt commun, expression(s) de dynamisme et d'adaptabilité aux mutations internes et externes (J.P. Houssel, ibid). Ces critères admettent bien entendu des soutiens ou incitations, qu'ils émanent de l'Etat, de Régions, de Comités d'expansion ou d'autres grands acteurs, ainsi que des extensions ou précisions (rôle des trames circulatoires, de réseaux économiques - filières, systèmes productifs -, importance des approches intégratrices, - globalisation -, interdépendance des champs de cohésion, rôle des acteurs « médiateurs »...).

\section{B. A partir de là, les constats relèvent de multiples « familles » de territoires aujourd'hui apparents :}

\section{Les territoires « performants »}

19 Sous l'angle de la performance économique, certains sont avancés grâce à l'innovation et à la technologie, gages d'adaptabilités : les «métropoles-technopoles », les « milieux innovateurs » (D. Maillat), les «districts industriels", les "systèmes productifs locaux »... En fait, il en est de relativement anciens : Choletais, Roannais, vallée de l'Arve, Haut-Jura, Haut Bugey, districts horlogers savoyards et comtois, pays de Montbéliard, et de plus récents (ceux de l'aéro-spatiale du Sud Ouest, de l'électromécanique - Trégor -, Bas-Dauphiné) ou repositionnés (Oyonnax: plasturgie, Thionvillois : mécanique avancée,...), d'organisés par ou en fonction de grandes firmes (Pays de Montbéliard et Pays de Besançon, et plus largement Rhin-Sud, Toulousain...) ou d'un tissu de PME (Choletais, Arve). Mais les territoires métropolitains sont euxmêmes fragmentés et leur dynamique davantage résiliaire et externalisée, liée par l'économie plus que par l'organisation socio-spatiale interne. Une assez grande diversité d'articulations entre bases économiques et systèmes sociaux locaux est visible dans les autres cas : de la simple « culture industrielle » au sein des familles des salariés - ou de la transmission des savoir-faire - à l'osmose entre entreprises et communautés -, aux imbrications de leurs réseaux, en passant par la formation et les voies du transfert technologique (CRITT ${ }^{11}$, pôles de compétences, IUT, ...). L'institutionnel public y intervient, par-delà ce dernier registre, par les équipements territoriaux nécessaires.

\section{Les territoires transformés ou en transformation}

Les recompositions démographiques (structures, flux), économiques (changements des systèmes de production agricole, essaimages ou relais dans l'industrie, tertiarisation, tourisme...), l'intervention de relais sociaux plus dynamiques, la promotion d'intercommunalité de projet et de contrats de développement, etc. en sont des facteurs majeurs: Monts du Lyonnais, Val de Lorraine de part et d'autre de Pont-àMousson, pays du Val de Loire (orbite parisienne), Pays du Mont Blanc ou côtes vendéenne ou basque (tourisme) en sont des exemples. Les pays de la Saône et du Rhône moyen illustrent pour leur part le rôle que peuvent jouer les axes de transports très équipés dans la recomposition (logistique, localisation d'industries, tourisme) ainsi 
que le renforcement des villes moyennes dans leurs fonctions et leurs acteurs (N. Commerçon).

Plus généralement, et notamment dans les " espaces intermédiaires ", des capacités de maintien des cohésions territoriales dépendent des rôles entretenus, élargis, adaptés des villes moyennes en réponse aux attentes de leurs campagnes (emplois, services, animation de la vie sociale, intermédiation économique, connexions aux réseaux) : Pays de Haguenau, de Sarrebourg, Barrois dans le Nord-Est, de Cognac, de Saintes et d'autres en Poitou-Charentes (J. Soumagne); leur efficacité peut dépendre aussi de leurs aptitudes à constituer des "réseaux de villes » - dont l'éclosion récente et concrétisée va au-delà du «label $»^{12}$, et à modeler les perspectives d'élargissement et/ou de complémentarité des territoires. Par contre, si manque le niveau de ces villes «moyennes» ou «de transition $»^{13}$, les bourgs et petites villes ont bien du mal à contrecarrer des écartèlements et les forces dissipatives internes et externes : ainsi, dans les marges Alsace du NW- Est Lorrain, entre Pau et Tarbes, en Basse Normandie orientale...

Les territoires tombant dans des aires urbanisées importantes sont encore plus transformés : centres locaux «déclassés " et "dépendants»; déficience accrue des logiques d'ordre spatial (dispersions, segmentations); fragilisation des structures sociales préexistantes - même dans des «bastions » de cohérence rurale qui résistent difficilement, tel le Kochesberg aux portes de Strasbourg - interférences conflictuelles d'enjeux et d'acteurs économiques, sociaux, spatiaux... Des «re-constructions » s'y imposent, mais tardent à se concrétiser.

\section{Les territoires gravement fragilisés}

On les trouve dans les espaces du "rural profond ", démographiquement dévitalisés, vieillis, passant à l'agriculture extensive et aux déprises foncières, au repli des bourgs, des PME..., et sous l'influence des «consommations" du "tourisme de nature». R. Chapuis et alii (op. cit. note 8) en décrivent les traits et les problèmes en Bourgogne, J.-P. Houssel ceux du Haut Vivarais. Les exemples pourraient être multipliés.

On les trouve aussi dans les territoires industriels durement frappés: pays noir de Saint-Etienne-Firminy, bassin sidérurgique lorrain, vallées textiles vosgiennes, ou de la houille blanche dans les Alpes; Valenciennois-Douaisis... Les actions de soutien aux reconversions et à l'emploi, pour importantes qu'elles aient été et soient encore, répondent "dans l'urgence », multiplient les intervenants publics et privés, nationaux et plus locaux - parfois concurrents -, restent principalement «industrielles » et exogènes, trop peu liées «territorialement » à d'autres volets (habitat, articulations à l'endogène socio-économique) ${ }^{14}$. Ainsi des césures nouvelles se créent, telles celles qui fragmentent le Bassin sidérurgique lorrain avec un «Pays haut " sinistré, un versant oriental bénéficiant mieux d'ouverture sur l'axe mosellan et une aire relancée par le Pôle Européen de Développement de Longwy, ou celles de vallées montagnardes entre amont et aval, etc.

\section{Les « faux territoires » (G. Di Méo, J.P. Jambes) et les systèmes politiques territorialisés}

Sont ainsi désignés des territoires suscités par des opportunismes d'élus, par des « logiques de guichet » au regard des systèmes d'aides et incitations. On peut certes en 
écarter certaines unités rassemblées dans des "contrats de pays " vivants des années 1974 et suivantes ou des structures de développement local, d'aménagement (SDAU, parcs naturels régionaux, chartes intercommunales ayant ces finalités); voire d'autres calées sur des objectifs moins globaux (Pays d'accueil touristique, comités de bassin d'emploi) à forte mobilisation endogène. Mais nombreux sont les cas de "récupération" de mémoires de "territorialités d'antan", "pays» de cadrages cantonaux (souvent obsolètes), de découpages «ad hoc » à la mesure du "poids » et (de) la « couleur» des leaders politiques des pays (notables locaux ou "parachutés ») (cf. Di Méo, op. cit.), de constructions à trop faible fonctionnalité ou assises participatives et démocratiques, encore qu'il y ait quelques réussites de mobilisation sociale, culturelle et identitaire (ex. de la Soule -ibid-) davantage que de développement économique.

\section{Parmi les faiblesses, se dégagent souvent :}

- l'excès de localisme, surtout en " pays ruraux d'enracinés » (J. Renard, ibid.), de repli sur le culturel passéiste, de rivalités locales, que reflètent bien les dimensions restreintes des actuelles communautés de communes ou (districts). Ainsi le Massif vosgien en compte 42, dont 20 de moins de 10000 hab. (1997), avec des sous-ensembles où elles sont encore absentes; la Vendée est partagée en 19 pays, les Mauges entre une dizaine de communautés de communes. Aucune région n'est exempte de ces défauts, ni de ces confusions induites entre "pays" et "bassins de vie", dont les structures restent en-deçà de "seuils de viabilité " (population, ressources financières, adéquations à des bassins d'habitat, à des réseaux d'entrepreneurs ou entre emploi et formation pour le développement local).

27 - des positionnements trop spécifiques, insuffisamment "globaux » : promotion de produits « du terroir », ou du tourisme ; intercommunalité de seule gestion, ou alliant trop peu aménagement et développement : ceci au gré de groupes ou acteurs leaders et par déficit de synergies d'objectifs ou d'intermédiation, par le jeu des interventions sectorisées...

28 - un primat de fonctionnalité, de cadrages préétablis (souvent inducteurs de hiatus), sur l'émergence d'un «état d'esprit » ou d'une « intelligence spécifique » (P. Gonin, op. cit. note 10), de vues globales ou intégratrices des différents maillons et articulations en chaînes prévalant dans l'aménagement et dans le développement ou en systèmes de cohésion, tous impliquant des dynamiques à ne pas figer.

- un déficit de lisibilité ou d'appropriation collective, imputable au moins en partie à celui de démocratie directe.

La liste n'est pas close...

\section{Pourtant divers champs d'opportunité se précisent, malgré quelques menaces ou inquiétudes.}

31 Les instances nationales et régionales s'investissent dans l'établissement de " territoires pertinents ", certes encore objets de débats et réflexions prospectives (de la Datar, des collectivités locales). Les soutiens institutionnels, par voies de contractualisations nombreuses, tentent de susciter coordinations et projets. La 
décentralisation pousse à développer les "gouvernances" associant les impératifs d'unité et de respect des diversités ${ }^{15}$.

A l'heure de l'affranchissement des distances et des accès commodes à l'information en temps réel par les NTIC, des systèmes d'information géographique et des réseaux multiples, se généralise la connaissance des expériences, des ingénieries, des innovations aidant à sortir d'attitudes passives ou repliées. Tandis que les sociétés «réinvestissent» les cadres de vie, les patrimoines, les territoires comme facteurs identitaires, tandis que l'économie se repositionne au regard des territoires et des potentiels extra-économiques, des réseaux de coordination interorganisationnelle (cf. coûts des transactions, théories de la confiance et des conventions), des chances nouvelles s'ouvrent pour des voies de restructuration efficiente de cohésions territoriales. S'y ajoutent les volontés de meilleures coordinations d'interventions territorialisées des politiques sectorielles (Régions et départements, Etat, Union européenne).

Cependant, force est de constater que jusqu'ici ces orientations n'ont guère réduit les écarts de dynamisme et d'aisance, ni atténué sensiblement la tendance aux fragmentations spatiales (espaces toujours plus "mosaïques»). Que subsistent les multiplicités et enchevêtrements des actions sectorielles, des contractualisations et zonages, avec des temporalités non harmonisées. Que s'amplifient les financements croisés et les chevauchements de compétences qui «plombent » maints projets. Une autre inquiétude est liée aux affaiblissements des composantes sociales de cohésion à plusieurs niveaux (familiales et intergénérationnelles, aux lieux de travail, dans les relations interterritoriales). En France, plus encore que dans le «modèle rhénan » où elles sont aussi menacées, leur délitage introduit des menaces dont les territoires risquent de faire les frais.

\section{Conclusion}

34 Ainsi, nombre de travaux récents aidant, par leurs convergences ou leurs complémentarités, à éclairer problématiques et réflexions sur le thème des "territoires", leurs articulations et leur examen (embrassant passé, présent et prospective), permettent d'insister sur l'importance des territoires et sur leurs positionnements « dans des chaînages ». Les territoires sont tout à la fois les maillons essentiels et fragiles dans une démarche d'ancrage amélioré de la géographie à l'aménagement-développement, qui en France est passé de l'aménagement du territoire à l'aménagement des territoires.

\section{NOTES}

1. Ex. ATP du CNRS : Observation du changement social, nombreuses publications entre 1980 et 1986. Bailly et alii (1998) Les concepts de la géographie humaine, $4^{\mathrm{e}}$ éd. rev. et augm. Colin. Brunet R. (1990) : «Evaluation et prospective des territoires », in Confér. sur la géographie. Min. Rech. et 
Technol. p. 25-44 : Inst. de la Décentralisation (1996) : La décentralisation en France. Datar (1994). Némery J.C. et alii : Le renouveau de l'aménagement du territoire en France et en Europe, Economica. Eckert D. (1996) : Evaluation et prospective des territoires, Reclus - Doc. Fr., Frémont et alii (1984) Géographie sociale, Masson. Duran P. (1999), Penser l'action publique, LGDJ. Greffe X. (1993) Sociétés post-industrielles et redéveloppement, Hachette. Groud H. et alii (1999) Mutations du service public et territoires, L'Harmattan. Glon E. et alii: Mutations économiques et recompositions des territoires, in IFRESI (1996) : Actes du Colloque «Changement régional et dynamique des territoires ». Lacour C. et Puissant S. (1987) : La tectonique des territoires, IERSO - Datar. Némery J.C., Wachter S. et alii (1994) Gouverner les territoires, Datar, éd. de l'Aube. Veltz P. (1996) Mondialisation, villes et territoires, PUF. Nombreux articles de revues: Hommes et Terres du Nord; Rev. d'Econ. Rég. et Urb.; Sc. de la Société (depuis 1990).

2. Nonn H. (2000) Réflexions sur les «territoires »: de l'intérêt de l'interdisciplinarité », in Carnets de la Maison des Sciences de l'Homme de Strasbourg (à paraître aux PUS).

3. Claverie J.C. : «Les cadres spatiaux de la vie de relation dans le Sud-Ouest de la France durant la première moitié du XIX ${ }^{\mathrm{e}}$ siècle ", Rev. Géogr. de l'Est 1973/3. E. Juillard (1974) : La "région", contributions à une géographie générale des espaces régionaux. Ophrys. Pr. Univ. de Strasbourg, p. 63-102.

4. Savy M., Veltz P. et alii (1993) Les nouveaux espaces de l'entreprise; et : (1995) Economie globale et réinvention du local, Datar. Ed. Aube. Léo P. et Philippe J. : «Tertiarisation des métropoles et centralité » RERU, 1998/1; Puissant S. : La métropolisation en question » RERU, 1997/4. Veltz P. (1996), op. cité en note 1).

5. Nombreuses publications : dont: $n^{\circ} 8$ Hors série de Sciences humaines (1995) et $n^{\circ} 2440$ de « Problèmes économiques» (1995). Benko G. et Lipietz A. (1992) Les régions qui gagnent, PUF. Ratti R. «L'espace régional actif », RERU 1997/4.

6. Laborie J.P., Langumier J.F., de Roo P. (1986) La politique française d'aménagement du territoire de 1950 à 1985. Doc. Fr. Rapports annuels d'activité de la Datar; situation actuelle décrite par J. Auroux (1998) : Réforme des zonages et aménagement du territoire. Rapport au $1^{\mathrm{er}}$ trimestre (multigr.).

7. Davezies L. (1996) Un essai de mesure de la dépendance des territoires français aux fonds publics. Publ. CEIL - Paris $12^{e}$ et «Le poids des fonds publics dans le revenu des villes et des régions » in : Pumain D. et Mattei M.F. (1998) Données urbaines 2, Anthropos, p. 329-342.

8. Dupuy C. et Gilly J.P. (1947) : Midi-Pyrénées : dynamisme industriel et renouveau rural (Doc. Fr.) ; Guibourdenche H., Marcou et alii (1997) L'Ardèche et la Drôme: activités, territoires et politique (Doc. Fr.). Chapuis R. et alii (1993) La Bourgogne, réalités et perspectives (multigr.) et « Le réseau urbain bourguignon ", in RGE 1994/2 ; H. Nonn (1999) Villes et aménagement régional en Alsace (Doc. Fr.) sont cités à titre d'exemples. Coll. (2001) : RITMA, Regard(s) croisé(s) sur les territoires de marge(s), collection la Maison des Sciences de l'Homme de Strasbourg, àparaître aux Presses Universitaires de Strasbourg (étude desmarges Moselle-Alsace du Nord Ouest et de l'ensemble Rhin-Sud).

9. Ce type d'analyse s'inspire d'un canevas devenu préalable à la prospective (Analyse AFOM), tel que la préparation du Schéma de développement de l'Europe communautaire (SDEC) le présente dans le premier projet officiel Noordjwijk, (1997).

10. Rolland-May C. (1996) : "Contribution à la définition du concept de 'pays' : application aux 'pays barrois' RGE 1996/3-4 ; et (1999) Dynamique et recomposition des territoires... Mosella 1999/3-4 (v. ibid, art. de S. de Ruffray). Nº spéc. Hommes et terres du Nord (1996/2) Les habits neufs des pays (art. de H. Nonn, JP. Houssel, J. Renard, G. Di Méo et J.P. Jambes, P. Bruyelle et P. Gonin) ; Norois 1999/1 Pays et développement local (art. de J. Renard, D. Guillermet, C. Rolland-May, J. Soumagne...). Baudelle G. et alii (1995) De l'intercommunalité au pays, IAAT. Ed. Aube. B. Kayser et alii (1993) Naissances de nouvelles campagnes, Datar, éd. Aube. IFRESI-LGH Lille 1 (1996) Pays, bassins de vie et discontinuités, Etudes $\mathrm{N}^{\circ} 13$ (multigr.). Kotas M. (1998) Politique de pays, Doc. Fr., Guigou J.L. (1996) Une ambition pour le territoire, Datar Ed. Aube. $\mathrm{N}^{\circ}$ spéc. « Espaces ruraux », RERU,1996/2 (art. de J. Cavailhes, H. Jayet, B. Ganne et N. Bertrand, X. Greffe...). 
11. CRITT $=$ Centre Régional d'Innovation de Transfert de Technologie.

12. Club national des réseaux de villes (1995) : Présentation et actions des réseaux de villes (fiches) et publication de leurs rencontres annuelles.

13. N. Commerçon et alii (1999) Villes de transition. Anthropos.

14. Grosdidier (1996) La reconversion, une ardente obligation. Doc. Fr..

15. Leca J.: «Gouvernance et institutions publiques: l'Etat entre sociétés nationales et globalisation » in : De Foucauld J.P. et alii (1996) La France en prospectives, O. Jacob. Calame P. et Talmant A. : «Un impératif catégorique de la gouvernance : concilier l'unité et la diversité », in Coll. (1997) L'Etat au cœur, ed. Desclées de Brouwer. Van Eeckhout L., Colloque de Cerisy (1999) Regards croisés sur la ville, Ed. Aube.

\section{RÉSUMÉS}

Les territoires sont des maillons à la fois essentiels et fragiles dans les organisations humaines. A partir de nombreux travaux effectués dans plusieurs disciplines qui se rencontrent sur ce concept et les thématiques qui s'y rattachent, l'article retient quelques grands moments de recompositions territoriales dans l'espace français des deux derniers siècles, ainsi que quelques familles de situations actuelles. On s'est efforcé en particulier de relever les interdépendances entre facteurs économiques, sociaux, géographiques et institutionnels intervenant dans leur constitution, leurs évolutions, leurs éléments de cohésion, bref, dans leurs dynamiques. Facteurs au regard desquels s'apprécient les forces et faiblesses, les perspectives favorables ou inquiétantes des territoires, dont l'évaluation constitue une solide contribution de la géographie à l'aménagement-développement.

Territories are at once essential and fragile links into human organisations. This article uses numerous works from the many disciplines focused on this concept to review some major examples of french territorial recomposition of the last two centuries, and some similar current situations. In particular an attempt will be made to pick out the interdependence between economic, social, geographical and institutional factors involved in their constitution, evolution, and cohesion, in short, their dynamics. It is the evaluation of the strengths and weaknesses, the positive and negative influences of these factors, that constitutes a robust contribution of Geography to the subject of development.

Die Territorien sind grundlegende und zugleich empfindliche Glieder menschlicher Organisationen. Ausgehend von zahlreichen, von mehreren Disziplinen geleisteten Arbeiten, die sich in Konzept und Thematik berühren, hält der Artikel einige grosse Augenblicke territorialer Neuordnung im französischen Raum der letzten beiden Jahrhunderte wie auch aktueller Situationen fest. Insbesondere sollen die Wechselbeziehungen zwischen ökonomischen, sozialen, geographischen und institutionellen Faktoren sichtbar gemacht werden, die in ihrer Konstitution, Entwicklung und Kohäsion, kurz in ihrer Dynamik zusammenwirken. Im Blick auf diese Faktoren können die Stärken und Schwächen, die günstigen oder beunruhigenden Perspektiven der Territorien ermessen werden, deren Bewertung einen soliden Beitrag der Geographie zur Raumordnung und -entwicklung darstellt. 
INDEX

Mots-clés : aménagement, France, recomposition, territoire, XIXe siècle, XXe siècle

Schlüsselwörter : 19 Jahrhundert, 20 Jahrhundert, Frankreich, Neuordnung, Raumordnung, Territorium

Keywords : 19th Century, 20th Century, development, France, recomposition, territory

\section{AUTEUR}

\section{HENRI NONN}

Professeur honoraire - Université Louis Pasteur - 3, rue de l'Argonne 67000 Strasbourg 